\title{
New Sub-Phenotyping of Subjects at High Risk of Type 2 Diabetes: What Are the Potential Clinical Implications?
}

\author{
Djordje S. Popovic (D) · Manfredi Rizzo · Edita Stokic • \\ Nikolaos Papanas
}

Received: March 25, 2021 / Accepted: April 20, 2021 / Published online: May 4, 2021

(c) The Author(s) 2021

\begin{abstract}
Prediabetes is defined as a condition of abnormal glucose metabolism, characterised by plasma glucose above normal range but not as high as required for the diagnosis of diabetes mellitus (DM). It represents a heterogeneous entity of intermediate glucose metabolism, including impaired fasting glucose, impaired glucose tolerance, and borderline glycated haemoglobin. Prediabetes is being increasingly recognised as an important metabolic state not
\end{abstract}

D. S. Popovic $(\bowtie) \cdot$ E. Stokic

Clinic for Endocrinology, Diabetes and Metabolic

Disorders, Clinical Center of Vojvodina, Novi Sad,

Serbia

e-mail: djordje.popovic@mf.uns.ac.rs

D. S. Popovic $\cdot$ E. Stokic

Medical Faculty, University of Novi Sad, Novi Sad, Serbia

M. Rizzo

Department of Health Promotion, Mother and Child Care, Internal Medicine and Medical Specialties of Internal Medicine (Promise), School of Medicine, University of Palermo, Palermo, Italy

\section{N. Papanas}

Diabetes Centre, Second Department of Internal

Medicine, Democritus University of Thrace,

University Hospital of Alexandroupolis,

Alexandroupolis, Greece only predisposing to a higher probability of future progression to DM, but also to an increased risk of different micro- and macrovascular complications. The recently proposed sub-phenotyping of individuals at increased risk of type $2 \mathrm{DM}$, which distinguishes six different clusters, offers the opportunity for the improvement in screening, prevention, and treatment algorithms. Such progress should also enable more efficient and cost-effective strategies aimed at decreasing the disease burden associated with prediabetes.

Keywords: Cardiovascular disease; Diabetes mellitus; Kidney disease; Precision medicine; Prediabetes; Type 2 diabetes mellitus 


\section{Key Summary Points}

Prediabetes is defined as a condition of abnormal glucose metabolism, characterised by plasma glucose above normal range but not as high as required for the diagnosis of diabetes mellitus.

Prediabetes is recognised as an important metabolic state predisposing to a higher probability of future progression to diabetes mellitus and to an increased risk of different micro- and macrovascular complications.

The recently proposed sub-phenotyping of individuals at increased risk of type 2 diabetes mellitus offers the opportunity for improved screening, prevention, and treatment algorithms.

This novel clustering should enable more efficient and cost-effective strategies aimed at decreasing the disease burden associated with prediabetes.

\section{DIGITAL FEATURES}

This article is published with digital features, including a summary slide, to facilitate understanding of the article. To view digital features for this article go to https://doi.org/10.6084/ m9.figshare.14447841.

\section{COMMENTARY}

Prediabetes is defined as a condition of abnormal glucose metabolism: plasma glucose (PG) is above the normal range but not as high as required for the diagnosis of diabetes mellitus (DM) [1]. According to current American Diabetes Association guidelines [2], diagnosis of prediabetes is based on the measurements of fasting PG (FPG), 2-h PG during a 75-g oral glucose tolerance test (OGTT), and glycated haemoglobin $\left(\mathrm{HbA}_{1 \mathrm{c}}\right)$. Prediabetes represents a heterogeneous entity of intermediate glucose metabolism abnormalities, including impaired fasting glucose $\quad(F P G \geq 5.6 \mathrm{mmol} / \mathrm{l}$ and $<7.0 \mathrm{mmol} / \mathrm{l}$ ), impaired glucose tolerance ( 2 -h PG $\geq 7.8 \mathrm{mmol} / \mathrm{l}$ and $<11.1 \mathrm{mmol} / \mathrm{l})$, and as borderline $\mathrm{HbA}_{1 \mathrm{c}}(\geq 5.7 \%$ and $<6.5 \%$ ) [2].

Prediabetes is increasingly being recognised as an important metabolic state predisposing to type 2 DM (T2DM), chronic diabetic complications, and cardiovascular diseases $[3,4]$. The main mechanisms underlying the transition from normal glucose tolerance (NGT) to prediabetes include increased insulin resistance (IR) and reduced pancreatic $\beta$-cell function $[5,6]$. Other perturbations are now known to include lipolysis, glucagon secretion, and decreased incretin effect $[5,6]$.

The prevalence of prediabetes varies according to the diagnostic criteria and the populations studied [7]. However, there is agreement that this prevalence is on the rise, suggesting that effective screening and timely interventions will be required [7]. In general, only 10\% of subjects with prediabetes progress to T2DM each year [3]. Others remain in the same intermediate hyperglycaemic state during lifespan, or even reverse to NGT $[3,8]$. Accordingly, we need an adequate tool to identify subjects at high risk of future T2DM and/or vascular complications.

Of note, DM may be much more heterogeneous than usually appreciated, and so Ahlqvist et al. [9] attempted to make a refined classification of adult-onset diabetes subgroups and their association with outcomes, with the aims to provide a useful tool for individualised treatment and to identify subjects with increased risk of complications [9]. Glutamate decarboxylase antibodies (GADA), age at diagnosis, body mass index (BMI), $\mathrm{HbA}_{1 \mathrm{c}}, \beta$-cell function and IR (using homoeostasis model assessment [HOMA] 2 estimates [HOMA2-B and HOMA2-IR] based on C-peptide concentration) are the six parameters evaluated [9]. Based on these, five different clusters have been defined [9]: cluster 1, with early-onset disease, relatively low BMI, poor metabolic control, insulin deficiency, and presence of GADA, marked as severe autoimmune diabetes; cluster 2 , with low age at onset, relatively low BMI, low insulin secretion, 
poor metabolic control, and absence of GADA, marked as severe insulin-deficient diabetes; cluster 3, with IR and high BMI, marked as severe insulin-resistant diabetes; cluster 4, with obesity but no IR, marked as mild obesityrelated diabetes; cluster 5 , characterised by older age and modest metabolic derangements, marked as mild age-related diabetes. Of note, based on prospective data on the development of complications from patient records, subjects in cluster 3 exhibit significantly higher risk of diabetic kidney disease than those in clusters 4 and 5 [9]; subjects in cluster 2 exhibited the highest risk of retinopathy [9]. This sub-stratification of DM has been recognised as a promising first step in the development of precision medicine in DM [8].

In a similar fashion, Wagner et al. [10] have recently identified six distinct clusters of subphenotypes of individuals who are at increased risk for T2DM. The initial clustering and identification of sub-phenotypes was enabled by using data of 899 participants at increased risk of DM (history of prediabetes, family history of $\mathrm{DM}, \mathrm{BMI}>27 \mathrm{~kg} / \mathrm{m}^{2}$, or history of gestational diabetes) from the Tuebingen Family study and Tuebingen Lifestyle Program study [10]. Analysis was based on data for preselected phenotyping variables including glucose challenge, insulin sensitivity and insulin secretion (using AUC0-30 C-peptide/AUC0-30 glucose and the Matsuda index, both derived from 2-h OGTT), high-density lipoprotein (HDL) cholesterol, liver fat content (assessed by ${ }^{1} \mathrm{HMR}$-spectroscopy), subcutaneous and visceral fat volume (using magnetic resonance tomography-based measurement), and a polygenic risk score for T2DM risk (63 of the top 94 diabetes-related genetic variants shown in one of the large-scale genome-wide association studies [11] were genotyped) [10]. The clustering was replicated among 6810 individuals from the occupational Whitehall II cohort using similar variables, including glycaemia during glucose challenge, insulin sensitivity and insulin secretion (calculated from glucose and insulin values at fasting and at $120 \mathrm{~min}$ of a 2 -h OGTT), fasting insulin, fasting triglycerides, waist circumference, hip circumference, BMI, and HDL cholesterol $[10,12]$. In the longitudinal analysis, all subjects with available data were followed for the development of diabetes, nephropathy, cardiovascular endpoints, and all-cause mortality.

Based on metabolic characteristics and risk profiles, the following six sub-phenotypes have been identified: cluster 1 , including individuals at low risk; cluster 2, including individuals at very low risk; cluster 3 , including individuals characterised by $\beta$-cell failure; cluster 4, including obese individuals at low risk; cluster 5 , including individuals characterised by IR and fatty liver who are at high risk; cluster 6, including individuals characterised bya high amount of visceral fat and an increased risk of nephropathy who are at high risk [10].

Shortly, according to data from the longitudinal analysis, patients in clusters 1,2 , and 4 have a low risk of developing DM and low mortality compared with those in the other three clusters: most subjects in cluster 2 were slim and exhibited a very low risk of developing diabetes complications [10]. Subjects in cluster 4 , although overweight, had relatively healthy metabolisms, due to storage of body fat in subcutaneous depots; conversely, subjects in cluster 3 had genetic risk factors for DM (higher frequency of the diabetes-associated G-allele of MTNR1B rs10830963 compared with cluster 1) and were characterised by poor insulin secretion and moderately elevated visceral fat compartments [10]. Although their mortality risk is moderate, they had a high risk of developing DM, cardiovascular complications, and kidney disease [9]. In cluster 5, the hallmarks were high fat liver content and severe IR, high risks of DM, cardiovascular complications, and nephropathy, and a relatively higher mortality rate [10]. Finally, subjects in cluster 6 were characterised by a high level of visceral fat, especially in the kidneys, and although their risk of DM was relatively low compared to those among clusters 3 and 5, their risks of nephropathy and mortality were high; cluster 6 exhibits IR, but lower liver fat content and higher insulin secretion compared with cluster 5 [10]. Additionally, the pathophysiological classification of diabetes-related genetic variants [13] suggested a lower presence of $\beta$-cell-related risk alleles in cluster 6 [10]. 
What, then, does this very novel classification contribute in practice? Considering that subjects in clusters 1, 2 and 4 have a low risk of developing DM and low mortality, it seems reasonable only to monitor them as often as their level of risk dictates and to make proper management of clinical and laboratory parameters. By contrast, subjects in clusters 3, 5, and 6 require immediate interventional measures. Importantly, aggressive lifestyle interventions are necessary in clusters 3 and 5 [14]. As one step forward, an appropriate pharmacological therapy might be considered in selected prediabetes subjects $[15,16]$, particularly with the use of agents with proven cardio-renal benefit in T2DM, such as glucagon-like peptide- 1 receptor agonists and sodium-glucose cotransporter-2 inhibitors, in order to provide an effective prevention of chronic complications and to assure a long healthy life to such subjects [17-19]. The need to protect kidney function appears especially important in cluster 6 , while the need to prevent cardiovascular complications appears especially important in clusters 3 and 5 [10] (Fig. 1).

The important limitation of the new classification is the lack of ethnic diversity of the investigated populations analysed in the study, and therefore such findings may only be applicable to white European populations. Also, in routine practice it may be not easy to distinguish some of the proposed clusters by using simple and widely available clinical and laboratory data. This imposes a need for further studies verifying this classification using more basic anthropometric and laboratory parameters. As far as applied interventions are concerned, it is still questionable how to potentially monitor and measure their effects. What should the specific aims and outcomes be, especially if the intervention involves usage of pharmacologic treatments? Future trials assessing the effectiveness of different therapeutic measures

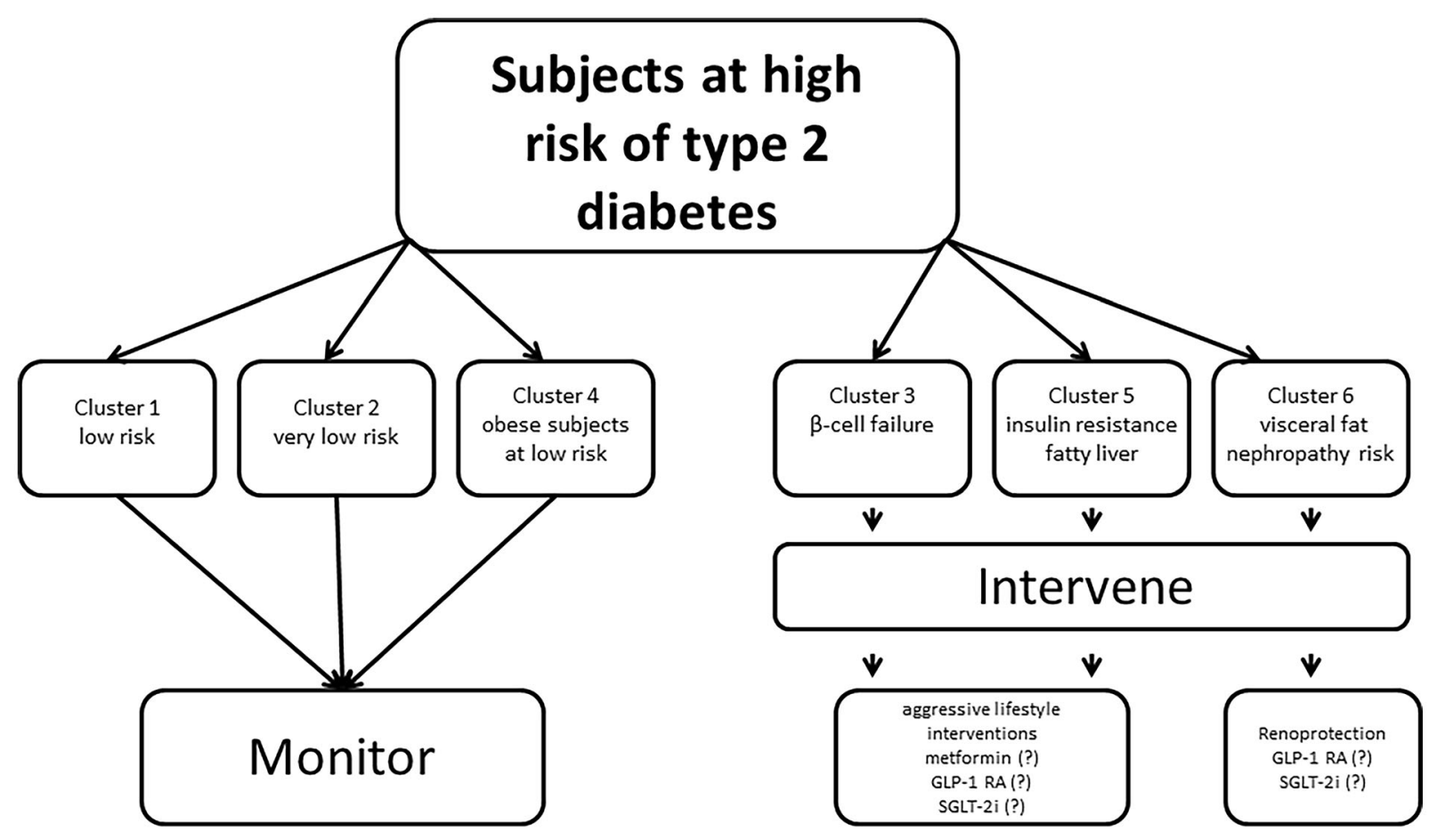

GLP-1 RA: glucagon-like peptide-1 receptor agonists SGLT-2i: sodium-glucose cotransporter-2 inhibitors

Fig. 1 Suggested approaches among different clusters of subjects at high risk of type 2 diabetes mellitus 
among different clusters should elucidate these ambiguities.

However, this interesting clustering brings clinicians a new tool for precise risk assessment among very heterogeneous groups of individuals at increased risk of T2DM. This should allow a timely identification of those in need of immediate intervention and selection of an appropriate therapeutic approach. It should also enable the avoidance of a burden on the health system associated with excessive measures aimed at those at low risk, although their transition to some of the high risk clusters could occur over the course of time. Anyway, this novel clustering of subjects at increased risk of T2DM undoubtedly represents a solid foundation for procreation of precision medicine also in prediabetes.

In conclusion, the recently proposed subphenotyping of individuals at increased risk of T2DM potentially allows much more precise cardiometabolic profiling and offers improvements in screening, prevention, and treatment algorithms. Such progress should also enable more efficient and cost-effective strategies aimed at decreasing the disease burden associated with prediabetes. This is of particular importance during the prolonged pandemic we are still facing caused by the novel severe acute respiratory syndrome coronavirus 2 (SARS-CoV2) infection, and its related diseases, coronavirus disease 2019 (COVID-19), since it is of crucial importance to keep subjects at risk of T2DM under proper control and prevent cardiorenal-metabolic complications, with diabetes being a main comorbidity associated with more severe forms of COVID-19 and associated mortality [20-22]. Future studies should address the potential association of different clusters of both diabetes and prediabetes with incidence and severity of COVID-19.

\section{ACKNOWLEDGEMENTS}

Authorship. All named authors meet the International Committee of Medical Journal Editors (ICMJE) criteria for authorship for this article, take responsibility for the integrity of the work as a whole, and have given their approval for this version to be published.

Author Contributions. Djordje S. Popovic analysed the literature and wrote the manuscript; Manfredi Rizzo reviewed and edited the manuscript; Edita Stokic reviewed and edited the manuscript; Nikolaos Papanas reviewed, edited, and finalised the manuscript.

Funding. No funding or sponsorship was received for this study or publication of this article.

Disclosures. This manuscript was written independently and the authors did not receive financial or professional help for its preparation. Djordje S. Popovic declares associations that might represent the conflict of interest with the following companies: Abbott, Alkaloid, AstraZeneca, Boehringer-Ingelheim, BerlinChemie, Eli Lilly, Galenika, Krka, Merck, Novo Nordisk, PharmaSwiss, Sanofi-Aventis, Servier, and Worwag Pharma. Manfredi Rizzo is Professor of Internal Medicine at University of Palermo, Italy, and currently Medical Director in Novo Nordisk Eastern Europe; he has given lectures, received honoraria and research support, and participated in conferences, advisory boards and clinical trials sponsored by many pharmaceutical companies including Amgen, AstraZeneca, Boehringer Ingelheim, Kowa, Eli Lilly, Meda, Mylan, Merck Sharp \& Dohme, Novo Nordisk, Novartis, Roche Diagnostics, Sanofi, and Servier. Edita Stokic declares associations that might represent a conflict of interest with the following companies: AstraZeneca, Boehringer-Ingelheim, Berlin-Chemie, Eli Lilly, Galenika, Hemofarm, Merck, MSD, Mylan, Novo Nordisk, PharmaSwiss, Proton System, and Sanofi-Aventis. Nikolaos Papanas has been an advisory board member of TrigoCare International, Abbott, AstraZeneca, Elpen, MSD, Novartis, Novo Nordisk, Sanofi-Aventis, and Takeda; has participated in sponsored studies by Eli Lilly, MSD, Novo Nordisk, Novartis, and Sanofi-Aventis; received honoraria as a speaker for AstraZeneca, Boehringer Ingelheim, Eli Lilly, Elpen, Galenica, MSD, 
Mylan, Novartis, Novo Nordisk, Pfizer, SanofiAventis, Takeda, and Vianex; and attended conferences sponsored by TrigoCare International, AstraZeneca, Boehringer Ingelheim, Eli Lilly, Novartis, Novo Nordisk, Pfizer, and SanofiAventis. None of the above-mentioned companies had any role in this article, which has been written independently, without any financial or professional help, and reflects only the opinion of the authors, without any role of the industry.

Compliance with ethics guidelines. This article is based on previously conducted studies and does not contain any novel data from studies with human participants or animals performed by any of the authors.

Data availability. Data sharing is not applicable to this article as no datasets were generated or analysed during the current study.

Open Access. This article is licensed under a Creative Commons Attribution-NonCommercial 4.0 International License, which permits any non-commercial use, sharing, adaptation, distribution and reproduction in any medium or format, as long as you give appropriate credit to the original author(s) and the source, provide a link to the Creative Commons licence, and indicate if changes were made. The images or other third party material in this article are included in the article's Creative Commons licence, unless indicated otherwise in a credit line to the material. If material is not included in the article's Creative Commons licence and your intended use is not permitted by statutory regulation or exceeds the permitted use, you will need to obtain permission directly from the copyright holder. To view a copy of this licence, visit http://creativecommons.org/licenses/by$\mathrm{nc} / 4.0 /$.

\section{REFERENCES}

1. Rett K, Gottwald-Hostalek U. Understanding prediabetes: definition, prevalence, burden and treatment options for an emerging disease. Curr Med Res Opin. 2019;35:1529-34.
2. American Diabetes Association. Classification and diagnosis of diabetes: standards of medical care in diabetes-2021. Diabetes Care. 2021;44(Suppl 1): S15-33.

3. Tabak AG, Herder C, Rathmann W, Brunner EJ, Kivimaki M. Prediabetes: a high-risk state for diabetes development. Lancet. 2012;379:2279-90.

4. Vas PRJ, Alberti KG, Edmonds ME. Prediabetes: moving away from a glucocentric definition. Lancet Diabetes Endocrinol. 2017;5:848-9.

5. Weyer C, Bogardus C, Mott DM, Pratley RE. The natural history of insulin secretory dysfunction and insulin resistance in the pathogenesis of type 2 diabetes mellitus. J Clin Invest. 1999;104:787-94.

6. Brannick B, Dagogo-Jack S. Prediabetes and cardiovascular disease: pathophysiology and interventions for prevention and risk reduction. Endocrinol Metab Clin North Am. 2018;47:33-50.

7. Hostalek U. Global epidemiology of prediabetespresent and future perspectives. Clin Diabetes Endocrinol. 2019;5:5.

8. Nathan DM, Davidson MB, DeFronzo RA, et al. Impaired fasting glucose and impaired glucose tolerance: implications for care. Diabetes Care. 2007;30:753-9.

9. Ahlqvist E, Storm P, Karajamaki A, et al. Novel subgroups of adult-onset diabetes and their association with outcomes: a data-driven cluster analysis of six variables. Lancet Diabetes Endocrinol. 2018;6: 361-9.

10. Wagner R, Heni M, Tabak AG, et al. Pathophysiology-based subphenotyping of individuals at elevated risk for type 2 diabetes. Nat Med. 2021;27: 49-57.

11. Mahajan A, Wessel J, Willems SM, et al. Refining the accuracy of validated target identification through coding variant fine-mapping in type 2 diabetes. Nat Genet. 2018;50:559-71.

12. Marmot M, Brunner E. Cohort profile: the Whitehall II study. Int J Epidemiol. 2005;34:251-6.

13. Udler MS, Kim J, von Grotthuss M, et al. Type 2 diabetes genetic loci informed by multi-trait associations point to disease mechanisms and subtypes: a soft clustering analysis. PLoS Med. 2018;15: e1002654.

14. Stoica RA, Diaconu CC, Rizzo M, et al. Weight loss programmes using low carbohydrate diets to control the cardiovascular risk in adolescents. Exp Ther Med. 2021;21:90. 
15. Crandall JP, Knowler WC, Kahn SE, et al. The prevention of type 2 diabetes. Nat Clin Pract Endocrinol Metab. 2008;4:382-93.

16. American Diabetes Association. Pharmacologic approaches to glycemic treatment: standards of medical care in diabetes-2021. Diabetes Care. 2021;44(Suppl 1):S111-24.

17. Pafili K, Rizzo M, Papanas N. New antihyperglycaemic agents and cardiovascular disease: let's be optimistic. Curr Opin Cardiol. 2018;33:444-54.

18. Sachinidis A, Nikolic D, Stoian AP, et al. Cardiovascular outcomes trials with incretin-based medications: a critical review of data available on GLP-1 receptor agonists and DPP-4 inhibitors. Metabolism. 2020;111:154343.
19. Maranta F, Cianfanelli L, Rizzo M, Cianflone D. Filling the gap between guidelines and real world in the cardiovascular approach to the diabetic patients: the need for a call to action. Int J Cardiol. 2021;329:205-7.

20. Ceriello A, Stoian AP, Rizzo M. COVID-19 and diabetes management: What should be considered? Diabetes Res Clin Pract. 2020;163:108151.

21. Stoian AP, Papanas N, Prazny M, Rizvi AA, Rizzo M. Incretin-based therapies role in COVID-19 era: evolving insights. J Cardiovasc Pharmacol Ther. 2020;25:494-6.

22. Papachristou S, Stamatiou I, Stoian AP, Papanas N. New-onset diabetes in COVID-19: time to frame its fearful symmetry. Diabetes Ther. 2021;12:461-4. 